\title{
Die Einzelstreuung schneller Elektronen über kleine Winkel
}

\author{
Von Gerhard Schulze-Pillot * und Walther Bothe \\ Aus dem Physikalischen Institut der Universität Heidelberg \\ und dem Institut für Physik im Max-Planck-Institut für medizinische Forschung \\ (Z. Naturforschg. 5 a, 440-447 [1950]; eingegangen am 21. Juli 1950)
}

In Fortsetzung früher unterbrochener Versuche wurden Absolutmessungen über Einzelstreuung von Elektronen von $1-2,5 \mathrm{MeV}$ im Bereich kleiner Streuwinkel $\left(13^{\circ}-25^{\circ}\right)$ an Polystyrol, Al, Ni, Ag und Au durchgeführt.

Die homogenen Primärelektronen wurden mit Rn-Präparaten und einem Linsenmonochromator hergestellt. Die Streuanordnung war ebenfalls zylindersymmetrisch.

Die Ergebnisse stimmen innerhalb $10 \%$ mit der Theorie von Mott überein.

Die Ursachen für die Abweichungen in den früher mitgeteilten vorläufigen Ergebnissen wurden aufgeklärt.

1. Die Einzelstreuung schneller Elektronen an Atomkernen gehört zu den Fundamentalproblemen der Teilchenphysik. Ihre Gesetzmäßigkeiten ergeben sich zwangsläufig aus der Diracschen Theorie des Elektrons ${ }^{1}$, sofern dabei der Kern als strukturlose Punktladung angesehen werden kann. Die zahlreichen experimentellen Untersuchungen über diesen Gegenstand liefern immer noch kein einheitliches Bild hinsichtlich der Übereinstimmung mit der Theorie. Die Schwierigkeit exakter Einzelstreumessungen liegt darin, daß man, um die Vielfachstreuung genügend weit herabzudrücken, mit sehr dünnen Streufolien und entsprechend geringen Streuintensitäten arbeiten muß, so daß schon sehr schwache Störeffekte die Ergebnisse merklich verfälschen können. Störstrahlungen werden vorzugsweise in der Nähe des Primärstrahls auftreten, so daß Messungen bei kleinen Streuwinkeln, um die es sich im folgenden immer handelt, besonders schwierig sind.

Die folgende Arbeit knüpft an eine Untersuchung von $\mathrm{B}$ ot he und $\mathrm{R}$ a tze $\mathrm{I}^{2}$ an. In dieser wurde statt der früher meist üblichen Nebelkammermethode die Geigersche Zählmethode auf das Problem angewandt. Dabei wurde eine axialsymmetrische Anordnung benutzt, die besonders für Messungen bei kleinen Streuwinkeln geeignet ist. Die Messungen wurden an Aluminium und Nickel im Energiebereich von 1 bis 2,5 $\mathrm{MeV}$ und im Winkelbereich von $\mathrm{rd} .13^{\circ}-25^{\circ}$

* Diss. Heidelberg. Vorgetragen auf der Tagung der Physikalischen Gesellschaft Württemberg-Baden-Pfalz in Karlsruhe am 9. Juni 1950.

1 N. F. M o t t, Proc. Roy. Soc. [London], Ser. A 124, 425 [1929]; 135, 429 [1931].

2 W. B othe u. L. Ratze l, Z. Physik 115, 497 [1940] (im folgenden als I zitiert). durchgeführt. Die Ergebnisse stimmten bei den kleineren Energien leidlich mit der Theorie überein, während bei größeren Energien namentlich das Aluminium Abweichungen bis $\mathrm{zu}+80 \%$ zu zeigen schien. Wegen des Krieges mußte diese Untersuchung vorzeitig abgebrochen werden.

Inzwischen haben $\mathrm{van} d \mathrm{e} \mathrm{Gra}$ aff und Mitarbb. ${ }^{3,4}$, nach einer Ionisationsmethode mit sehr starker Strahlenquelle (elektrostatischer Generator) ausgedehnte Streumessungen in demselben Energiebereich und bei Winkeln von $20^{\circ}-50^{\circ}$ durchgeführt. Diese ergaben zunächst ${ }^{3}$ beim Aluminium Abweichungen ähnlicher Art wie bei Bothe und Ratzel. Die Abweichungen verschwanden aber bei Fortführung der Untersuchung ${ }^{4}$, und die Theorie konnte durchgehend für alle untersuchten Elemente mit einer Genauigkeit von etwa $10 \%$ bestätigt werden.

Im folgenden soll nun über die Fortführung und den Abschluß der Arbeit von Bothe und Ratzel berichtet werden. Die Messungen wurden in folgenden Punkten verbessert. Es gelang, den Nulleffekt herabzusetzen. Andererseits standen auch stärkere Strahlenquellen zur Verfügung. Dadurch war intensitätsmäßig die Möglichkeit gegeben, die zu messenden Strahlen stark zu filtern, um weichere Störstrahlungen sicher auszuschalten. Die Korrektionen für überlagerte Vielfachstreuung, die in I etwas unterschätzt worden waren, konnten auf Grund neuerer Arbeiten genauer ermittelt werden. Die Messungen wurden ferner auf

3 R. J. Van de Graaff, W. W. Buechner u. H. F e s h b a ch, Physic. Rev. 69, 452 [1946].

4 W. W. B uechner, R. J. Van de Graaff, A. Sperduto, E. A. Burril u. H. Feshbach, Physic. Rev. 72, 678 [1947]. 


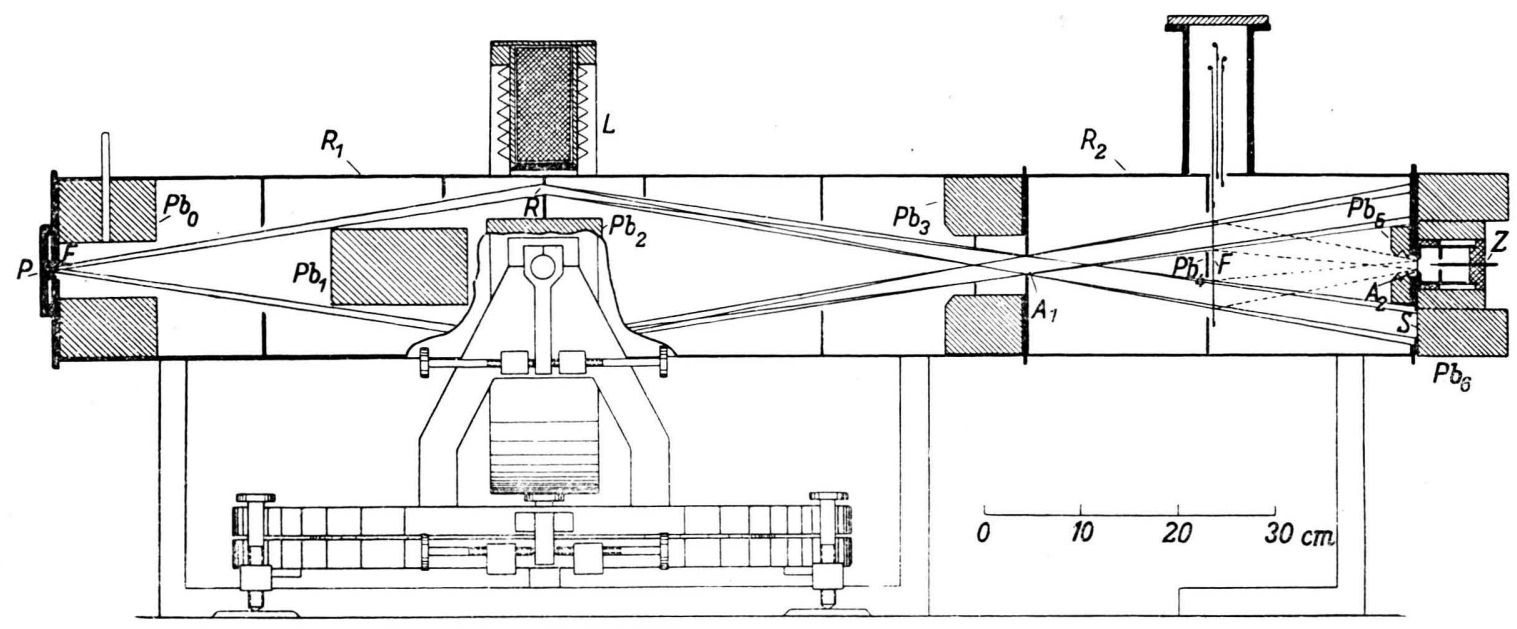

Abb. 1. Versuchsanordnung.

das ganze periodische System ausgedehnt. Schließlich ermöglichten noch einige inzwischen erschienene numerische Auswertungen der theoretischen Streuformel einen exakteren Vergleich mit der Theorie. Als Gesamtergebnis sei vorausgenommen, daß keine außerhalb der Meßfehler liegenden Abweichungen von der Theorie gefunden wurden.

\section{Versuchsanordnung}

2. Monochromator und Streukammer. Die allgemeine Anordnung ist in I ausführlich beschrieben. Sie ist in Abb. 1 nochmals dargestellt.

Aus dem Rn-Präparat $\mathbf{P}$ treten inhomogene $\beta$-Strahlen in den $100 \mathrm{~cm}$ langen Monochromator $\mathrm{R}_{1}$ mit der magnetischen Elektronenlinse L ein. Der Strahlengang wird definiert durch die Ringblende $\mathrm{R}$ mit den Radien 7,5 und $8,5 \mathrm{~cm}$ und die Austrittsblende $\mathrm{A}_{1}$ von $2 \mathrm{~cm}$ Durchmesser. Das aus $A_{1}$ austretende hohlkegelförmige Bündel, dessen Impulsbreite etwa $10 \%$ beträgt, trifft in der Mitte der $40 \mathrm{~cm}$ langen Streukammer $R_{2}$ auf die auswechselbare Streufolie F. Die durch die sekundäre Austrittsblende $A_{2}$ in den Spitzenzähler Z gestreuten Elektronen werden gezählt. Das Zählerfenster, das den austretenden Strahl begrenzt, hat $1 \mathrm{~cm}$ Durchmesser. Das Eintrittsfenster E und das Austrittsfenster $\mathrm{A}_{2}$ sind mit Glimmer von 3 bzw. $5 \mathrm{mg} / \mathrm{cm}^{2}$ verschlossen, das Zählerfenster mit $3,5 \mathrm{mg} / \mathrm{cm}^{2}$ Glimmer plus $1,5 \mathrm{mg} / \mathrm{cm}^{2}$ Aluminium.

Die Primärintensität und ihre radiale Verteilung werden mit demselben Zähler längs der radialen Schlitzblende S gemessen, die ebenfalls mit Glimmer von $5 \mathrm{mg} / \mathrm{cm}^{2}$ verschlossen ist. Die Schlitzblende befindet sich in einem zweiten, leicht auswechselbaren Abschlußdeckel. An diesem Deckel sind zur sicheren Führung zwei Schienen angebracht, zwischen denen der Zähler, in einer Pertinaxplatte gehalten, an dem Schlitz entlanggeschoben wird. Eine Blende von $4 \mathrm{~mm}$ Durchmesser definiert das ausgezählte Flächenstück. Das Verhältnis dieser Fläche zur Gesamtfläche des Zählerfensters wurde mit einem schwachen $\beta$-Präparat kontrolliert. Auf der Schlitzblende entspricht jedem Radius ein praktisch einheitlicher Wert des Streuwinkels. Bei der Messung der Streuintensität in $\mathrm{A}_{2}$ wird daher über einen Streuwinkelbereich von rd. $13^{\circ}-25^{\circ}$ gemittelt. Das Innere der Apparatur ist vollständig mit Cellophan ausgekleidet, um die Rückdiffusion von ungewollter Streustrahlung tunlichst zu unterbinden. Die verbleibende Rückdiffusion ist mit großen Energieverlusten verbunden 5 . Die auf diese Weise entstehende Störstrahlung kann daher durch Filter unschädlich gemacht werden.
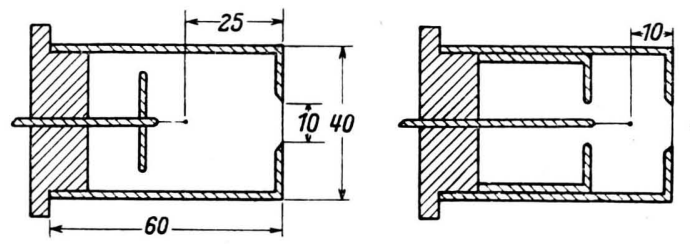

Abb. 2. Spitzenzähler, alte und neue Form.

Die einzige wesentliche Änderung an diesem Teil der Apparatur bestand darin, daß außer den Bleikörpern $\mathrm{Pb}_{1}-\mathrm{Pb}_{6}$ als weiterer Schutz gegen die $\gamma$-Strahlung der Quelle noch ein Bleiring $\mathrm{Pb}_{0}$ hinter der Eintrittsblende angebracht wurde. Hierdurch wurde der Nulleffekt auf $1 / 3$ herabgedrückt. Während der Messung betrug der Druck in der Apparatur 10-4 Torr.

3. S p it z e n z ähler. Der früher verwendete Spitzenzähler wurde zur Verbesserung der Zähleigenschaften etwas verändert. Abb. 2 zeigt die beiden Formen nebeneinander. Um Nachentladungen zu vermeiden, wurde das Kügelchen näher an das Fenster herangesetzt. Damit bei diesem Abstand der Zähler gleichmäßig über die Fensterbreite anspricht, wurde der Wulst durch die mit dem Mantel verbundene Kreisringscheibe ersetzt. Durch die Scheibe werden praktisch alle Kraftlinien auf dem Kügel-

5 W. B o the, Z. Naturforschg. 4 a, 542 [1949]. 
chen zusammengedrängt. Der Nulleffekt nahm durch diese Änderung von 15 auf 20 Ausschläge pro min zu. Mit der Bleiabschirmung an der Apparatur betrug der Nulleffekt ohne Quelle nur noch rd. 6/min. Die Zählerfüllung bestand aus 12 Torr Argon +2 Torr Alkohol, die Zählspannung betrug $1300 \mathrm{~V}$.

Es war zu verlangen, daß der Zähler über die ganze Fläche des Fensters und bei Einfallswinkeln zwischen 0 und $13^{\circ}$ gleichmäßig auf die einfallenden Elektronen ansprach. Dies wurde mit einem ausgeblendeten $\beta$-Strahlbündel von $1 \mathrm{~mm}$ Durchmesser geprüft. Bei geeigneter Zählspannung wurde konstante Ansprechwahrscheinlichkeit über die ganze Fensterfläche bei Einfallswinkeln bis $\mathrm{zu} 25^{\circ}$ festgestellt, und zwar für einen Spannungsbereich von $60 \mathrm{~V}$.

4. V e r s t ä r k e r. Der Verstärker hat als Eingang eine Löschstufe, anschließend einen einstufigen Untersetzer und an diesen galvanisch angekoppelt die Endröhre. Die Löschstufe ist nach $\mathrm{Mai}$ a $\mathrm{r}-\mathrm{L}$ eibnitz ${ }^{6}$ als Multivibrator geschaltet. Da beim Spitzenzähler der Konstanzbereich erst $200 \mathrm{~V}$ über dem Einsatz beginnt, wurde ein Löschimpuls von mehr als $200 \mathrm{~V}$ gebraucht. Deshalb wurde die Anodenspannung der ersten Röhre durch $\mathrm{Zu}$ schalten einer Anodenbatterie auf $300 \mathrm{~V}$ erhöht. Die Untersetzerstufe ist nach $\mathrm{B}$ a ld in g e $\mathbf{r}^{\mathbf{7}}$ gebaut und in den Schaltelementen auf die verwendeten Röhren NF 2 zugepaßt. Die direkte Ankopplung der Endröhre liefert abwechselnd einen positiven und negativen Treppenimpuls zum Anziehen und Loslassen des Zählwerkes.

Als Zeitkonstante der Löschstufe wurde $1,5 \cdot 10^{-3}$ sec gewählt, um Nachentladungen ganz zu unterdrücken. Das Auflösungsvermögen der ganzen Anordnung betrug $4,5 \cdot 10^{-3} \mathrm{sec}$. Da nie mit mehr als 800 Stößen/min gearbeitet wurde, waren die Auflösekorrekturen klein und sicher zu berechnen.

5. Strahlenquelle. Es wurden ähnlich wie in I Rn-Hütchen als Strahlenquellen verwendet. Die Emanation wurde mit heißem Ca-Metall weitgehend gereinigt, so daß dauerhafte Quellen von $120 \mathrm{mC}$ verwendet werden konnten. Die $\beta$-Strahlen traten durch ein Glimmerfenster von $3-4 \mathrm{mg} / \mathrm{cm}^{2}$ aus.

6. Streufolien. Die Streufolien wurden von Messingrahmen getragen, die außen quadratisch $13 \times 13 \mathrm{~cm}$ waren und eine runde Öffnung von im allgemeinen $12 \mathrm{~cm}$ Durchmesser hatten. Für die Messungen an Kohlenstoff [Polystyrol $(\mathrm{CH})_{\mathrm{x}}$ ], Aluminium und Gold wurden käufliche Folien verwendet. Die Folien wurden auf chemische Reinheit geprüft*. Nickel- und Silberfolien wurden nach dem in I angegebenen Verfahren hergestellt durch Galvanisieren auf Kupfer und Ablösen des Kupfers in Kupferoxydammoniaklösung.

Da die galvanische Herstellung genügend großer und dünner Goldfolien auf Schwierigkeiten stieß, wurden käufliche Blattgoldfolien verwendet. Diese waren nur $8 \times 8 \mathrm{~cm}$ groß. Daher mußten in diesem Falle Rahmen von nur 7,8 cm Innendurchmesser benutzt werden, gegenüber $12 \mathrm{~cm}$ bei den anderen Substanzen. Von dem Primärstrahlkegel, dessen äußerer Durchmesser am Ort der

6 H. M a i e r-Leibnitz, Rev. Sci. Instr. 19, 500 [1948].

7 E. B a l d in g e r, Helv. Physica Acta 19, 202 [1946].
Folie $9 \mathrm{~cm}$ betrug, wurde dadurch der Außenteil abgeschnitten und der ursprüngliche Streuwinkelbereich von $13^{\circ}-25^{\circ}$ auf $13^{\circ}-22^{\circ}$ verkleinert.

Nach Beendigung einer Meßreihe wurden die Folien stückweise ausgewogen. Sie erwiesen sich als hinreichend homogen.

\section{Vorversuche}

7. Eichung des Monochromators. Die Eichung der Spule, die in I im unteren Energiebereich an den Linien des RaB durchgeführt worden war, wurde an diesen Linien wiederholt. Es ergaben sich auf 0,5\% genau dieselben Eichwerte, daher wurde die gesamte in I angegebene Eichkurve auch den neuen Messungen zugrundegelegt.

8. Nulleffekt. Dem Nulleffekt bei Anwesenheit der Quelle wurde besondere Aufmerksamkeit zugewendet, weil er bei der Auswertung der Messungen entscheidend eingeht. Mit eingesetzter Quelle betrug der Nulleffekt ein Mehrfaches des „natürlichen“ Nulleffekts. Dies konnte zweierlei Ursachen haben: die auf den Zähler wirkende $\gamma$-Strahlung der Quelle und vagabundierende Elektronen im Inneren der ganzen Apparatur. Die letztere Störstrahlung ist die gefährlichere, weil sich ihr Einfluß mit der Foliendicke und dem Magnetfeld ändern kann. Deshalb wurden folgende Versuche mit eingesetzter Quelle gemacht. Die Zahlenangaben beziehen sich auf eine Quellenstärke von $50 \mathrm{mC}$.

Ohne Magnetfeld und ohne Streufolie betrug der Nulleffekt rd. 50/min. Einbringen einer Streufolie erhöhte den Nulleffekt etwa proportional der Foliendicke, und zwar bis zu etwa $100 \%$ bei $4,5 \mathrm{mg} \mathrm{Al} / \mathrm{cm}^{2}$. Wurde jedoch durch $2 \mathrm{~mm}$ Blei über der Quelle die primäre $\beta$-Strahlung ferngehalten, so war der Nulleffekt unabhängig von der eingeschalteten Streufolie. Dieselbe Wirkung hatte ein $\mathrm{Cu}$ Filter von nur $0,28 \mathrm{~mm}$ vor dem Zähler. Der gesamte Nulleffekt betrug dann 49/min mit und ohne Streufolie. Diese Versuche lehrten, daß die Erhöhung des Nulleffektes, die ohne Filter mit Streufolie eintritt, auf mehrfach gestreute Primärelektronen zurückzuführen war. Diese konnten jedoch keine höhere Energie als $0,7 \mathrm{MeV}$ haben, da sie schon durch $0,28 \mathrm{~mm} \mathrm{Cu}$ vollständig absorbiert wurden. Da die Hauptmessungen nur bei höheren Energien und mit Filtern von mindestens $0,28 \mathrm{~mm} \mathrm{Cu}$ gemacht wurden, kann dabei diese Störstrahlung als ausgeschaltet gelten. Weiter zeigen diese Versuche, daß der von den $\gamma$-Strahlen verursachte Teil des Nulleffektes (43/min) unabhängig von der Dicke der Streufolie war.

Wurde nun ohne Streufolie und ohne Filter die Linse angeschaltet, so erhöhte sich wieder der Nulleffekt um rd. $30 \%$, praktisch unabhängig von der Erregung der Linsenspule in dem in Betracht kommenden Bereich. Diese Erhöhung konnte wiederum durch passend gewählte $\mathrm{Cu}$-Filter vor dem Zähler vollständig aufgehoben werden. Dieselbe Erscheinung trat in verstärktem Maße auf, wenn die Bleischeibe $\mathrm{Pb}_{4}$ (Abb.1) entfernt wurde. Hiernach handelte es sich sehr wahrscheinlich um eine Streustrahlung, die an den Rändern der Austrittsblende $\mathrm{A}_{1}$ des Monochromators entstand und durch Rückdiffusion

* Die Analyse wurde dankenswerterweise im Laboratorium der Badischen Anilin- und Sodaf a b rik AG., Ludwigshafen, ausgeführt. 
von den Wänden der Streukammer in den Zähler gelangte.

Die Gesamtheit dieser Versuche zeigte, daß der $\gamma$-Anteil des Nulleffektes unabhängig von der Dicke der Streufolie war, während der $\beta$-Anteil durch passend gewählte Filter vor dem Zähler unschädlich gemacht werden konnte und mußte. Dann konnte einfach der ohne Streufolie gemeșsene Nulleffekt verwendet werden.

9. F i l t e rver s u c h e. In den Hauptversuchen hatten die Cu-Filter vor dem Zähler Stärken von 0,28;0,36; 0,$50 ; 0,72 \mathrm{~mm}$ bezüglich für die Strahlenergien 0,$98 ; 1,41$; 1,$90 ; 2,44 \mathrm{MeV}$. Diese Dicken waren nach Ziff. 8 ausreichend, um die „wilden“ Streuelektronen auszuschalten. Sie waren so bemessen, daß sie Elektronen von $<60 \%$ der Primärenergie praktisch vollständig absorbierten, wie auch noch durch besondere Absorptionsversuche bestätigt wurde. Dieselben Filter wurden natürlich bei der Messung der Primärintensität wie auch der Streuintensität verwendet.

Für diese Filter wurde nun die Durchlässigkeit bei den zugehörigen Primärenergien gemessen, und zwar einmal im Primärbündel an der Schlitzblende S (Abb. 1), dann, nach Einbringen einer Streufolie, im Streubündel an der Austrittsblende $\mathbf{A}_{2}$. Im Primärbündel ergaben sich Durchlässigkeiten von rd. $40 \%$. Im Streubündel aber waren die gemessenen Durchlässigkeiten durchweg geringer. Der Unterschied betrug bis zu rd. 25\% und wurde mit zunehmender Primärenergie systematisch größer. Die Erklärung für das scheinbar geringere Durchdringungsvermögen der Streustrahlung liegt auf der Hand. Die nach Ziff. 8 festgestellte Störstrahlung trifft auch auf die ganze Streufolie und liefert so einen Beitrag zur gemessenen Streustrahlung. Wegen ihrer geringeren Energie wird sie jedoch wesentlich stärker durch die Folie gestreut als die eigentliche Primärstrahlung und täuscht daher ein geringeres Durchdringungsvermögen und eine $\mathrm{zu}$ hohe Intensität der einzelgestreuten Elektronen vor. Auf diese Weise kann ein im Verhältnis zur Primärintensität sehr kleiner Bruchteil an Störstrahlung schon eine merklich zu hohe Streuintensität vortäuschen, wenn man, wie in I, ohne Filter mißt. Hierin liegt offenbar eine der Ursachen für die abweichenden Ergebnisse von I; auch der Gang dieser Abweichungen mit der Energie findet damit seine Aufklärung.

\section{Hauptversuche und Meßergebnisse}

10. Die Primärintensität. Als Primärintensität $n_{\mathrm{p}}$ sei die gesamte pro min auf die Streufolie fallende Teilchenzahl definiert. Sie wurde, nachdem das für die Streumessung benutzte Rn-Präparat genügend weit abgeklungen war, an der Schlitzblende $\mathrm{S}$ gemessen, wie in Ziff. 2 beschrieben. Die Umrechnung auf gleiche-Intensität geschah nach der bekannten Halbwertszeit von 3,825 d.

Es sei $f_{\mathrm{p}}$ die Fläche der Zählerblende am Schlitz, $n(r)$ die Zahl der Teilchen, die pro min im Achsenabstand $r$ durch $f_{\mathrm{p}}$ treten. Dann ist

$$
n_{\mathrm{p}}=0,915 \frac{2 \pi}{f_{\mathrm{p}}} \int r n(r) d r .
$$

Der Faktor 0,915 trägt der Schattenwirkung der Haltestäbe an den Bleikörpern Rechnung, die sich geometrisch zu 8,5\% berechnet. Die gemessene Funktion

$$
r n(r)=N(r)
$$

ist in Abb. 3 für die verschiedenen Primärenergien aufgetragen. Die Kurven lassen sich, wie man sieht, durch Umrechnung auf gleiches Maximum gut zur Deckung bringen. Die ausgezogene Kurve gilt für den normalerweise verwendeten Folienrahmen von $12 \mathrm{~cm}$ Durchmesser, der das Primärbündel frei durch-

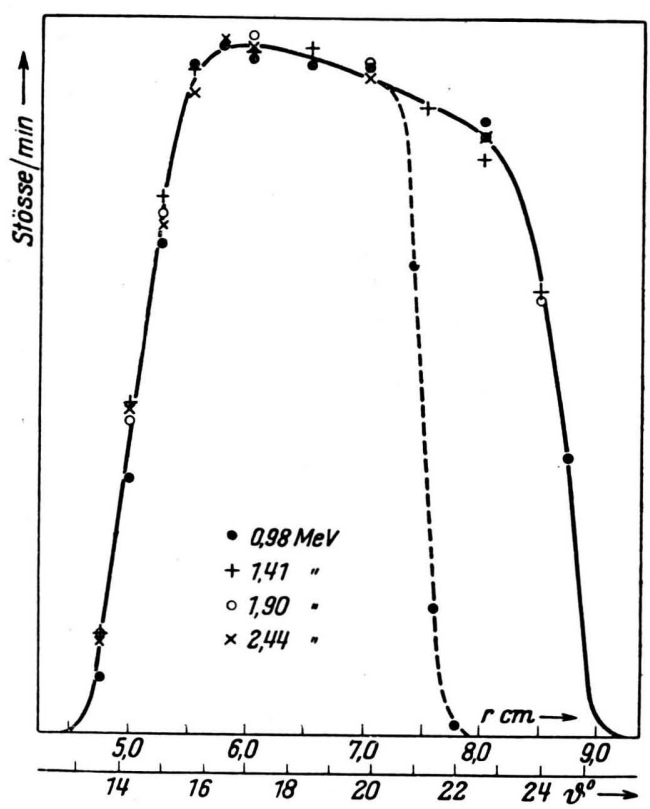

Abb. 3. Verteilung der Primärelektronen $N(r)$ an der Schlitzblende, relativ.

läßt. Für die Goldfolien mußten aus technischen Gründen (Ziff. 6) kleinere Rahmen von 7,8 cm Durchmesser verwendet werden, die das Primärbündel außen abschnitten. Für diese gilt die gestrichelte Kurve. Als Abszisse ist außer dem Radius der zugehörige Streuwinkel eingetragen.

Jeweils nach Abschluß einer Streumeß-Serie wurde die Primärverteilung an 3 Punkten kontrolliert. Die endgültigen Primärintensitäten sind in Tab. 1 zusammengestellt. Die Nulleffekte sind abgezogen.

11. Die Streuintensität. Der Nulleffekt wurde ohne Streufolie mit der gleichen Spulenstromstärke wie die Streuintensität gemessen. Die Streuintensität war durchweg nur von derselben Größenordnung wie der Nulleffekt, der bei $100 \mathrm{mC}$ mit Filter $91 / \mathrm{min}$ betrug. Bei $2,44 \mathrm{MeV}$ wurde vorsichts- 


\begin{tabular}{|c|c|c|c|c|}
\hline $\begin{array}{c}\text { Spulen- } \\
\text { strom } \\
\text { A }\end{array}$ & $\begin{array}{c}\text { Energie } \\
\text { MeV }\end{array}$ & $\begin{array}{c}\text { Filter } \\
\text { mm Cu }\end{array}$ & $\begin{array}{c}n_{\mathrm{p}} \cdot 10^{-6} \\
12 \mathrm{~cm}- \\
\text { Rahmen }\end{array}$ & $\begin{array}{c}n_{\mathrm{p}} \cdot 10^{-6} \\
7,8 \mathrm{~cm}- \\
\text { Rahmen }\end{array}$ \\
\hline 1,60 & 0,98 & 0,28 & 1,83 & 1,30 \\
2,20 & 1,41 & 0,36 & 1,07 & 0,76 \\
2,90 & 1,90 & 0,50 & 0,680 & 0,483 \\
3,70 & 2,44 & 0,72 & 0,328 & 0,233 \\
\hline
\end{tabular}

Tab. 1. Primärintensität, Stöße/min. $50 \mathrm{mC}$.

halber die Linse nach je 5 min wieder für $5-10 \mathrm{~min}$ abgeschaltet, um Überlastung $\mathrm{zu}$ vermeiden. So waren erhebliche Meßzeiten erforderlich, um den mittleren statistischen Fehler auf wenige Prozente herabzudrücken. Dabei waren die Folien so dünn gewählt, daß die Korrektion für Vielfachstreuung (Ziff. 12) nicht mehr als etwa - 15\% ausmachte.

Die Ergebnisse der endgültigen Streumessungen sind in Tab. 2, Spalte 1-4, zusammengestellt. $n_{\mathrm{s}}$ ist die Zahl der gestreuten Elektronen, die pro min und $50 \mathrm{mC}$ in das Zählerfenster von $1 \mathrm{~cm}$ Durchmesser eintraten. Aluminium wurde sowohl mit dem normalen Folienrahmen als auch mit dem kleineren Rahmen gemessen, der beim Gold verwendet wurde. Dies diente als Kontrolle dafür, daß der kleinere Rahmen keine neuen Fehlerquellen mit sich brachte, etwa durch Streustrahlung vom Rande.

Wie der Vergleich der Tab. 1 und 2 zeigt, ist der Bruchteil der auf die Folie fallenden Elektronen, der in den Zähler hineingestreut wird, nur von der Größenordnung $5 \cdot 10^{-5}$.

Einige systematische Messungen über die $\mathrm{Ab}$ hängigkeit der Streuintensität von der Schichtdicke bei $\mathrm{Al}$ sind in Abb. 4 dargestellt. Die oberen Punkte stellen den Quotienten aus der gemessenen Streuintensität und der Schichtdicke in willkürlichem Maßstab dar.

Entsprechende Messungen wie in Tab. 1 und 2 wurden für $\mathrm{Al}, \mathrm{Ni}$ und $\mathrm{Ag}$ auch ohne Filter, also wie in I durchgeführt. Hierauf wird in Ziff. 14 noch eingegangen werden.

Um schließlich noch den Einfluß der Filterung bei den leichtesten Elementen zu prüfen, wurden direkte Vergleichsmessungen zwischen Aluminium und Polystyrol bei der Energie 0,98 MeV durchgeführt, und zwar einmal mit besonders starker Filterung, dann ganz ohne Filter. Diese Messungen sind in Tab. 3 wiedergegeben.

8 C. T. Chase u. R. T. Cox, Physic. Rev. 58, 243 [1940].

9 Handbuch Physik XXII/2, S. 18 folg. [1933].

10 G. Groetz ing er, M. I. B e rger u. F. I. Ribe, Physic. Rev. 77, 584 [1950].

\section{Auswertung und Diskussion}

12. Korrektur für Vielfachstreuung. Der relative Zusatz $F$ an Vielfachstreuung wurde nach der von $\mathrm{Ch}$ a s e und $\mathrm{Cox}{ }^{8}$ angegebenen Formel berechnet:

$$
F=\overline{\varepsilon^{2}}\left(\sin ^{-2} \vartheta / 2-1 / 2\right) .
$$

$\vartheta$ bedeutet den Winkel, für den der Streuquerschnitt gemessen wird. Daher ist $\sin ^{-2} \vartheta / 2$ mit Berücksichtigung der Winkelabhängigkeit der Streuintensität zu mitteln. Diese ist für Rutherford-Verteilung durch $N(r) \sin ^{-4} \vartheta / 2$ gegeben, wo $\vartheta$ als Funktion von $r$ zu betrachten ist. In ausreichender Näherung kann man also in (3) setzen

$$
\sin ^{-2} \vartheta / 2=J_{6} / J_{4},
$$

wenn man zur Abkürzung schreibt

$$
J_{v}=\frac{\int N(r) \sin ^{-v} \vartheta / 2 d r}{\int N(r) d r} .
$$

Diese Ausdrücke wurden graphisch an Hand von Abb. 3 ermittelt.

$\overline{\varepsilon^{2}}$ bedeutet das mittlere Ablenkungsquadrat der Vielfachstreuung auf der mittleren Weglänge der um den Winkel $\vartheta$ abgelenkten Elektronen. Für diese Weglänge kann hier die Foliendicke selbst gesetzt werden, da sich beide um weniger als $1 \%$ unterscheiden.

Das mittlere Ablenkungsquadrat ist für eine GaußVerteilung $\overline{\varepsilon^{2}}=2 \lambda^{2}$, wobei $\lambda$, die wahrscheinlichste Ablenkung, näherungsweise berechnet werden kann nach $^{9}$

$$
\lambda=\frac{0,7}{V} \frac{V+0,511}{V+1,022} \sqrt{\left(Z^{2}+Z\right) \frac{\sigma}{A}} .
$$

Hierin ist $V$ die Strahlenergie in $\mathrm{MeV}, \sigma$ die Schichtdicke in $\mathrm{g} / \mathrm{cm}^{2}, A$ das Atomgewicht und $Z$ die Ordnungszahl der Streusubstanz. Der halbempirische Zahlenfaktor 0,7 ist nach neueren Ergebnissen ${ }^{10}$ an Stelle von 0,8 gesetzt worden. Der Beitrag der Atomelektronen ist unter der Wurzel berücksichtigt.

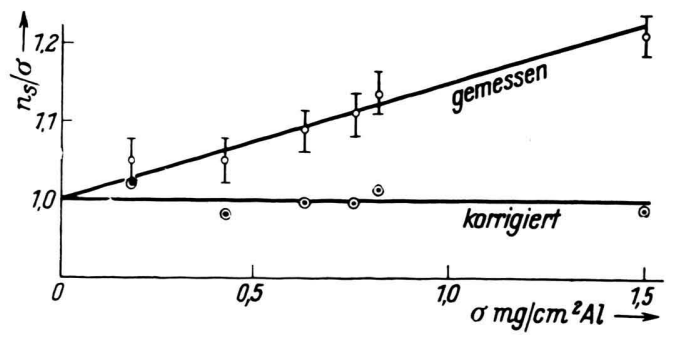

Abb. 4. Anstiegskurve von Aluminium bei $0,98 \mathrm{MeV}$. 


\begin{tabular}{|c|c|c|c|c|c|c|c|c|}
\hline 1 & 2 & 3 & 4 & 5 & 6 & 7 & 8 & 9 \\
\hline $\begin{array}{c}\text { Energie } \\
\mathrm{MeV}\end{array}$ & $\begin{array}{c}\text { Streu- } \\
\text { substanz } \\
\text { (Rahmẹ) }\end{array}$ & $\begin{array}{l}\text { Schicht- } \\
\text { dicke } \\
\mathrm{mg} / \mathrm{cm}^{2}\end{array}$ & $n_{\mathrm{s}}$ exp. & $\begin{array}{l}\text { Vielf. } \\
\text { korr. } \\
(1+F)^{-1}\end{array}$ & $\begin{array}{c}n_{\mathrm{s}} \exp \\
\text { korr. }\end{array}$ & $n_{\mathrm{s}}$ theor. & $\frac{n_{s} \text { exp. }}{n_{s} \text { theor. }}$ & $\begin{array}{l}\text { Mittl. } \\
\text { statist. } \\
\text { Fehler }\end{array}$ \\
\hline $\begin{array}{l}0,98 \\
1,41 \\
1,90 \\
2,41\end{array}$ & $\begin{array}{c}(\mathrm{CH})_{x} \\
(12 \mathrm{~cm})\end{array}$ & $\begin{array}{l}1,93 \\
4,00 \\
6,14 \\
8,07\end{array}$ & $\begin{array}{l}82,5 \\
54,0 \\
34,1 \\
13,4\end{array}$ & $\begin{array}{l}0,86 \\
0,85 \\
0,86 \\
0,88\end{array}$ & $\begin{array}{l}71,1 \\
46,2 \\
29,4 \\
11,7\end{array}$ & $\begin{array}{r}66,0 \\
43,5 \\
25,5 \\
10,4\end{array}$ & $\begin{array}{r}1,08 \\
1,06 \\
1,15 \\
1,12 \\
1,10\end{array}$ & $\begin{array}{l}0,04 \\
0,04 \\
0,05 \\
0,06 \\
0,03\end{array}$ \\
\hline $\begin{array}{l}0,98 \\
0,98 \\
1,41 \\
1,90 \\
1,90 \\
2,44 \\
2,44\end{array}$ & $\underset{(12 \mathrm{~cm})}{\mathrm{Al}}$ & $\begin{array}{l}0,59 \\
1,53 \\
1,53 \\
2,65 \\
2,70 \\
4,08 \\
5,86\end{array}$ & $\begin{array}{c}45,6 \\
141 \\
37,7 \\
27,2 \\
27,5 \\
13,6 \\
20,3\end{array}$ & $\begin{array}{l}0,91 \\
0,80 \\
0,88 \\
0,88 \\
0,88 \\
0,88 \\
0,83\end{array}$ & $\begin{array}{c}41,3 \\
113 \\
33,4 \\
23,8 \\
24,1 \\
11,8 \\
16,9\end{array}$ & $\begin{array}{c}41,3 \\
107 \\
33,8 \\
22,4 \\
22,8 \\
10,8 \\
15,5\end{array}$ & $\begin{array}{l}1,00 \\
1,05 \\
0,99 \\
1,06 \\
1,06 \\
1,09 \\
1,09 \\
1,05\end{array}$ & $\begin{array}{l}0,04 \\
0,04 \\
0,04 \\
0,05 \\
0,05 \\
0,06 \\
0,06 \\
0,02\end{array}$ \\
\hline $\begin{array}{l}0,98 \\
0,98 \\
1,41 \\
2,44\end{array}$ & $\begin{array}{c}\mathrm{Al} \\
(7,8 \mathrm{~cm})\end{array}$ & $\begin{array}{l}1,07 \\
1,32 \\
2,50 \\
5,86\end{array}$ & $\begin{array}{c}79,0 \\
105 \\
63,5 \\
17,5\end{array}$ & $\begin{array}{l}0,84 \\
0,81 \\
0,82 \\
0,83\end{array}$ & $\begin{array}{l}66,3 \\
85,2 \\
51,8 \\
14,7\end{array}$ & $\begin{array}{r}66,1 \\
82,0 \\
49,5 \\
13,7\end{array}$ & $\begin{array}{r}1,01 \\
1,04 \\
1,05 \\
1,06 \\
1,04\end{array}$ & $\begin{array}{l}0,03 \\
0,03 \\
0,04 \\
0,05 \\
0,02\end{array}$ \\
\hline $\begin{array}{l}0,98 \\
1,41 \\
1,41 \\
1,90 \\
1,90 \\
2,44\end{array}$ & $\begin{array}{c}\mathrm{Ni} \\
(12 \mathrm{~cm})\end{array}$ & $\begin{array}{l}0,545 \\
0,81 \\
1,00 \\
0,81 \\
1,00 \\
2,56\end{array}$ & $\begin{array}{l}96,0 \\
47,0 \\
47,2 \\
17,4 \\
20,3 \\
19,2\end{array}$ & $\begin{array}{l}0,84 \\
0,87 \\
0,85 \\
0,92 \\
0,90 \\
0,85\end{array}$ & $\begin{array}{l}80,5 \\
41,0 \\
40,0 \\
15,9 \\
18,3 \\
16,3\end{array}$ & $\begin{array}{r}82,0 \\
38,5 \\
47,5 \\
14,7 \\
18,1 \\
14,4 \\
M\end{array}$ & $\begin{array}{l}0,98 \\
1,06 \\
0,85 \\
1,08 \\
1,01 \\
1,13 \\
1,02\end{array}$ & $\begin{array}{l}0,04 \\
0,04 \\
0,04 \\
0,04 \\
0,05 \\
0,06 \\
0,02\end{array}$ \\
\hline $\begin{array}{l}0,98 \\
0,98 \\
1,41 \\
1,41 \\
1,90 \\
1,90 \\
2,44\end{array}$ & $\underset{(12 \mathrm{~cm})}{\mathrm{Ag}}$ & $\begin{array}{l}0,315 \\
0,450 \\
0,450 \\
0,605 \\
0,450 \\
0,605 \\
1,41\end{array}$ & $\begin{array}{c}82,5 \\
132 \\
35,0 \\
51,6 \\
14,9 \\
20,0 \\
15,6\end{array}$ & $\begin{array}{l}0,85 \\
0,81 \\
0,89 \\
0,86 \\
0,93 \\
0,91 \\
0,86\end{array}$ & $\begin{array}{c}70,0 \\
106 \\
31,1 \\
44,0 \\
13,8 \\
18,1 \\
13,4\end{array}$ & $\begin{array}{r}75,0 \\
107 \\
34,0 \\
45,6 \\
13,0 \\
17,5 \\
12,7\end{array}$ & $\begin{array}{l}0,93 \\
0,99 \\
0,91 \\
0,96 \\
1,06 \\
1,03 \\
1,05 \\
: \quad 0,99\end{array}$ & $\begin{array}{l}0,04 \\
0,04 \\
0,04 \\
0,05 \\
0,05 \\
0,05 \\
0,06 \\
0,02\end{array}$ \\
\hline $\begin{array}{l}0,98 \\
0,98 \\
1,41 \\
2,44\end{array}$ & $\underset{(7,8 \mathrm{~cm})}{\mathrm{Au}}$ & $\begin{array}{l}0,230 \\
0,225 \\
0,460 \\
0,90\end{array}$ & $\begin{array}{l}83,3 \\
81,8 \\
53,8 \\
12,5\end{array}$ & $\begin{array}{l}0,84 \\
0,85 \\
0,83 \\
0,86\end{array}$ & $\begin{array}{l}70,0 \\
69,0 \\
44,7 \\
10,8\end{array}$ & $\begin{array}{r}74,0 \\
72,5 \\
47,1 \\
11,0\end{array}$ & $\begin{array}{r}0,95 \\
0,95 \\
0,95 \\
0,98 \\
0,96\end{array}$ & $\begin{array}{l}0,04 \\
0,04 \\
0,04 \\
0,05 \\
0,02\end{array}$ \\
\hline
\end{tabular}

Tab. 2. Streuintensität, Stöße/min. $50 \mathrm{mC}$ und Vergleich mit der Theorie.

In Tab. 2 und 3 sind in Spalte 5 die an Spalte 4 anzubringenden Korrektionsfaktoren, und in Spalte 6 die korrigierten experimentellen Werte der Streuintensität aufgeführt.

Die in Abb. 4 wiedergegebenen Messungen über die Dickenabhängigkeit der Streuintensität wurden ebenfalls in der angegebenen Weise für Vielfachstreuung korrigiert. Wie man sieht, zeigen die korrigierten Werte exakte Proportionalität mit der
Schichtdicke. Dies beweist, daß die Korrektionen richtig berechnet wurden.

Die von Van de Graaff und Mitarbb. ${ }^{3}$ benutzte Formel für die in (3) eingehende mittlere quadratische Ablenkung nach einer Arbeit von Goudsmit und Saunderson ${ }^{11}$ führt zu dem gleichen Resultat.

11 S. Goudsmit u. J. L. S a unders on, Physic. Rev. 57, 24 [1940]; 58, 36 [1940]. 
13. Theoretische Formeln. Mott ${ }^{1}$ hat auf der Grundlage der Dirac-Gleichungen die Theorie der Kernstreuung schneller Elektronen entwickelt. Für den Streuquerschnitt ergibt sich ein sehr komplizierter Ausdruck, den man sich in eine Reihe nach Potenzen von $\alpha \mathrm{Z}$ entwickelt denken kann, wo $\alpha=1 / 137$ die Feinstrukturkonstante ist. Mott hat selbst eine Näherungsformel bis zur ersten Ordnung in $\alpha \mathrm{Z}$ angegeben. Eine etwas davon abweichende Formel haben $\mathrm{Sex} \mathrm{l}^{12}$ und $\mathrm{Urban}{ }^{13}$ mitgeteilt. $\mathrm{B}$ artlett und Wats on ${ }^{14}$ gaben die numerische Auswertung der strengen Mottschen Formel für die Ordnungszahl $Z=80$, und danach $\mathrm{McK}$ in ley und $\mathrm{Feshbach}{ }^{15}$ eine Reihenentwicklung nach $\alpha \mathrm{Z}$ bis

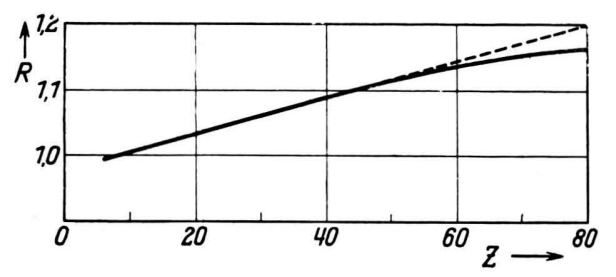

Abb. 5. Der wellenmechanische Korrektionsfaktor $R$ als Funktion von $Z$.

zur 4. Ordnung, die zusammen mit den Werten von Bartlett und Watson eine vollständige Berechnungsgrundlage des Streuquerschnittes für alle Ordnungszahlen liefert.

In der Arbeit von McKinley und Feshbach wird auch die Mottsche erste Näherung richtiggestellt. Sie lautet, als Verhältnis des wellenmechanischen Streuquerschnittes $\sigma$ zum klassischen Rutherford-Querschnitt $\sigma_{\mathrm{R}}$ geschrieben,

$$
\begin{gathered}
R=\sigma / \sigma_{\mathrm{R}}=1-\beta^{2} \sin ^{2} \vartheta / 2 \\
+\pi \beta Z \alpha \sin \vartheta / 2(1-\sin \vartheta / 2) .
\end{gathered}
$$

Bei Mott steht $\cos ^{2} \vartheta / 2$, bei Sexl-Urban 1 an Stelle von $1-\sin \vartheta / 2$.

In Abb. 5 sind die exakt berechneten $R$-Werte für 1,5 MeV, gemittelt über unseren Winkelbereich, als ausgezogene Kurve aufgetragen. Die 1. Näherung nach (7) wird durch die gestrichelte Gerade dargestellt. Man sieht, daß man nur einen Fehler von $+0,5$ bzw. $+3 \%$ begeht, wenn man auch für $\mathrm{Ag}$

12 Th. S e x l, Z. Physik 81, 178 [1933].

13 P. U r b a n, Z. Physik 119, 67 [1942].

14 J. B. Bartlett u. R. E. Watson, Proc. Amer. Acad. Arts. Sci. 74, 53 [1940].

15 W. A. McKinley u. H. Feshbach, Physic. Rev. 74, 1759 [1948]. und Au mit der Näherungsformel (7) rechnet. Für die anderen Energien gilt praktisch dasselbe. Daher kann man die theoretischen Streuintensitäten nach (7) berechnen und nachträglich bei $\mathrm{Ag}$ und $\mathrm{Au} \mathrm{0,5}$ bzw. 3\% abziehen.

Der Beitrag der Atomelektronen zur Streuintensität kann bei diesen kleinen Winkeln als das $Z^{-1}$ fache der Rutherford-Streuung angesetzt werden. Für eine Atomart und einen Streuwinkel würde also die Streuintensität sein:

$$
\begin{gathered}
n_{\mathrm{s}}=\frac{1}{4} n_{\mathrm{p}} \frac{L \sigma}{A}\left(\frac{Z e^{2}}{m c^{2}}\right)^{2} \frac{1-\beta^{2}}{\beta^{4}} \sin ^{-4} \vartheta / 2 \\
\cdot(R+1 / Z) d \Omega,
\end{gathered}
$$

wo $L$ die Loschmidtsche Zahl, $\sigma$ die Flächendichte der Folie ist. $d \Omega$ ist die Öffnung des ausgenutzten Streukegels, also $f_{\mathrm{s}} / a^{2}$, wenn $f_{\mathrm{s}}$ die Fläche des Zählerfensters $\left(\pi / 4 \mathrm{~cm}^{2}\right)$ und $a$ der Abstand StreufolieZählerfenster $(20 \mathrm{~cm})$ ist. Für unseren Fall eines kontinuierlichen Streuwinkelbereichs und einer aus verschiedenen Atomarten zusammengesetzten Streufolie (Polystyrol) ergibt sich aus (7) und (8) durch einfache Umrechnung mit Benutzung der Abkürzung (5)

$$
\begin{aligned}
n_{\mathrm{s}}= & \frac{1}{4} n_{\mathrm{p}} \frac{L \sigma}{\sum A}\left(\frac{e^{2}}{m c^{2}}\right)^{2} \frac{1-\beta^{2}}{\beta^{4}}\left[\sum\left(Z^{2}+Z\right) J_{4}\right. \\
& \left.-\beta^{2} \sum Z^{2} J_{2}+\pi \beta a \sum Z^{3}\left(J_{3}-J_{2}\right)\right] \frac{f_{\mathrm{s}}}{a^{2}} .
\end{aligned}
$$

Die Größen $J_{v}$ wurden wieder graphisch an Hand von Abb. 3 bestimmt. Die damit berechneten theoretischen Streuintensitäten mit den oben angegebenen kleinen Korrekturen für Ag und Au sind in Spalte 7 der Tab. 2 und 3 eingetragen. Spalte 8 gibt das Verhältnis der korrigierten experimentellen (Sp. 6) zu den theoretischen (Sp. 7) Streuintensitäten. In der letzten Spalte sind die berechneten statistischen Fehler dieses Verhältnisses eingetragen.

14. Diskus s i on. Die Spalte 8 der Tab. 2 zeigt keinen systematischen Gang mit der Strahlenergie. Die Energieabhängigkeit der Streuung wird also durch die Mottsche Theorie richtig wiedergegeben. Bildet man für jede Substanz den Mittelwert über alle Energien, so liegt die Abweichung von der theoretischen Streuintensität überall, mit Ausnahme des Polystyrols, innerhalb der statistischen Fehlergrenze. Es besteht also auch bezüglich der Materialabhängigkeit und der Absolutwerte Úbereinstimmung mit der Theorie.

$\mathrm{Zu}$ der kleinen Abweichung beim Polystyrol ist folgendes $\mathrm{zu}$ sagen. Durch magnetische Analyse 


\begin{tabular}{|c|c|c|c|c|c|c|c|c|}
\hline 1 & 2 & 3 & 4 & 5 & 6 & 7 & 8 & 9 \\
\hline $\begin{array}{c}\text { Energie } \\
\mathrm{MeV}\end{array}$ & $\begin{array}{c}\text { Streu- } \\
\text { substanz }\end{array}$ & $\begin{array}{l}\text { Schicht- } \\
\text { dicke } \\
\mathrm{mg} / \mathrm{cm}^{2}\end{array}$ & $n_{\mathrm{s}} \exp$ & $\begin{array}{l}\text { Vielf. } \\
\text { korr. } \\
(1+F)^{-1}\end{array}$ & $\begin{array}{c}n_{\mathrm{s}} \exp . \\
\text { korr. }\end{array}$ & $n_{\mathrm{s}}$ theor. & $\frac{n_{\mathrm{s}} \exp .}{n_{\mathrm{s}} \text { theor. }}$ & $\begin{array}{l}\text { Mittl. } \\
\text { statist. } \\
\text { Fehler }\end{array}$ \\
\hline \multicolumn{9}{|c|}{ a) gefiltert mit $0,36 \mathrm{~mm} \mathrm{Cu}$} \\
\hline $\begin{array}{l}0,98 \\
0,98\end{array}$ & $\begin{array}{c}(\mathrm{CH})_{\mathrm{x}} \\
\mathrm{Al} \\
(\mathrm{CH})_{\mathrm{x}}: \mathrm{Al}\end{array}$ & $\begin{array}{l}1,93 \\
1,55\end{array}$ & $\begin{array}{l}43,0 \\
77,0\end{array}$ & $\begin{array}{l}0,86 \\
0,80\end{array}$ & $\begin{array}{l}37,0 \\
61,5 \\
0,603\end{array}$ & 0,605 & 0,99 & 0,03 \\
\hline \multicolumn{9}{|c|}{ b) ungefiltert } \\
\hline $\begin{array}{l}0,98 \\
0,98\end{array}$ & $\begin{array}{c}(\mathrm{CH})_{\mathrm{x}} \\
\mathrm{Al} \\
(\mathrm{CH})_{\mathrm{x}}: \mathrm{Al}\end{array}$ & $\begin{array}{l}1,93 \\
1,55\end{array}$ & $\begin{array}{l}290 \\
467\end{array}$ & $\begin{array}{l}0,86 \\
0,80\end{array}$ & $\begin{array}{l}250 \\
\frac{374}{10,670}\end{array}$ & 0,605 & 1,11 & 0,015 \\
\hline
\end{tabular}

Tab. 3. Relativmessung, Polystrol : Aluminium.

einzelgestreuter Elektronen hat der eine von uns ${ }^{16}$ nachgewiesen, daß bei so leichtatomigen Stoffen selbst mit verhältnismäßig kleinen Schichtdicken noch Streuelektronen mit etwas verminderter Energie auftreten. Diese können in folgender Weise gedeutet werden. Durch Streuung an den Atomelektronen über größere Winkel entstehen in leichtatomigen Stoffen verhältnismäßig viele Elektronen mit herabgesetzter Energie, die deswegen bevorzugt zusätzliche Kernstreuung erfahren und so in den Zähler gelangen. Da die spektrale Intensität dieser Elektronen mit Annäherung an die Primärenergie steigt, sind sie schwer vollständig wegzufiltern. Aber der in Tab. 3 aufgeführte Versuch mit verstärktem Filter (0,36 statt $0,28 \mathrm{~mm} \mathrm{Cu}$ ) spricht dafür, daß in der Tat beim Polystyrol eine weichere Begleitstrahlung noch schwach mitgemessen wurde. Mit dem Filter ergibt sich auf 1\% das theoretische Verhältnis Polystyrol zu Aluminium, während ohne Filter die Abweichung $+11 \%$ beträgt.

Das Gesamtergebnis ist also, daß die Einzelstreuung von Elektronen von $1-2,5 \mathrm{MeV}$ im Streuwinkelbereich $13^{\circ}-25^{\circ}$ im ganzen periodischen System innerhalb von höchstens $10 \%$ mit der Mottschen Theorie übereinstimmt.

Daß für dieses Ergebnis die Filterung entscheidend war, zeigten die in Ziff. 11 schon erwähnten Messungen ohne Filter. Sie ergaben, in derselben Weise ausgewertet, Abweichungen von der Theorie, die bis zu $30 \%$ betrugen und mit wachsender Primärenergie zunahmen, ähnlich wie in I beobachtet.

Die vorliegenden Messungen sind bisher die einzigen, die bei so kleinen Streuwinkeln ausgeführt wurden. Sie bilden aber den Anschluß an die Messungen von Van de Graaff und Mitarbb., die sich auf Streuwinkel von $20^{\circ}-50^{\circ}$, teilweise bis $60^{\circ}$, in etwa demselben Energiebereich beziehen. Auch diese Autoren fanden Übereinstimmung mit der Theorie innerhalb etwa $10 \%$.

Dagegen scheint es sicher, daß bei größeren Streuwinkeln erhebliche Abweichungen von der Theorie auftreten können. Der eine von uns ${ }^{\mathbf{1 7}}$ hat bei $106^{\circ}$ für Elektronen von einigen $100 \mathrm{keV}$ die Z-Abhängigkeit der Einzelstreuung untersucht. Die Messungen müssen als verhältnismäßig sicher gelten, da die einzelgestreuten Elektronen noch magnetisch analysiert wurden. Es zeigten sich beträchtliche Abweichungen von dem theoretischen Verlauf.

Auf die sehr zahlreichen früheren Arbeiten, die mit der Nebelkammer oder mit inhomogenen Elektronen durchgeführt wurden, braucht hier nicht nochmals eingegangen $\mathrm{zu}$ werden, sie sind schon in 2 und in ${ }^{3}$ diskutiert. Meist ließen die dabei angewandten Methoden keine große Genauigkeit zu, weshalb auch die Ergebnisse sehr widerspruchsvoll waren.

16 W. B o th e, Z. Naturforschg. 4 a, 88 [1949].

17 W. B o th e, Z. Naturforschg. 5 a, 8 [1950]. 